K. Kessels et al., "Innovative Market Schemes for Integrated Multi-energy Systems," 2019 16th International Conference on the European Energy Market (EEM), Ljubljana, Slovenia, 2019, pp. 1-6, doi: 10.1109/EEM.2019.8916382. (accepted manuscript version)

Digital Object Identifier : 10.1109/EEM.2019.8916382

URL: https://ieeexplore.ieee.org/document/8916382

(c) 2019 IEEE. Personal use of this material is permitted. Permission from IEEE must be obtained for all other users, including reprinting/ republishing this material for advertising or promotional purposes, creating new collective works for resale or redistribution to servers or lists, or reuse of any copyrighted components of this work in other works. 


\section{Innovative Market Schemes for Integrated Multi-energy Systems}

\author{
Kris Kessels, Shahab Shariat Torbaghan, \\ Ana Virag, Hélène Le Cadre \\ VITO, Boeretang 200, \\ $2400 \mathrm{Mol}$, Belgium \\ EnergyVille, ThorPark 8310, \\ 3600 Genk, Belgium \\ Email: kris.kessels@vito.be
}

\author{
Guillaume Leclercq, Peter Sels, Mehdi Madani \\ N-SIDE, Boulevard Baudouin 1er, 25, \\ 1348 Louvain-La-Neuve, Belgium
}

\begin{abstract}
Multi-carrier energy systems create opportunities to decrease $\mathrm{CO}_{2}$ emissions, and improve the performance of energy systems by combining the benefits from different energy carriers. Currently, electricity, gas and heat systems are however economically decoupled in time by separate markets with different time frames and very different characteristics. This paper proposes five innovative multi-carrier market schemes which can better reflect the interactions between the different energy carriers, which eventually can lead to higher overall social welfare. The schemes range from a single carrier energy market scheme with separate, sequential day-ahead markets for different energy carriers to a unified multi-carrier energy market scheme with one unique multi-carrier market. Other schemes with single and/or multi-carrier markets at local and/or global level are also proposed. A qualitative evaluation of the five multi-carrier market schemes is given based on three indicators: economic efficiency, confidentiality level and resemblance to the current EU energy market designs.
\end{abstract}

\section{INTRODUCTION}

\section{A. Motivation and Background}

Multi energy systems (MES), in which different energy carriers interact with each other, have recently been receiving increasing attention from the scientific and regulatory communities. This concept represents an important opportunity to decrease $\mathrm{CO}_{2}$ emissions, and provides opportunities to improve the performance of the energy system by combining the benefits from the different energy carriers. Multi energy systems (e.g., energy systems with energy conversion technologies such as combined heat and power, gas turbine, heat pump, electric boilers, chillers, power to gas,...) already exist today [1], but most of these systems are currently characterized by relatively low penetration rates of conversion and storage technologies.

Today, the different energy vectors are largely operated in a decoupled, independent way with little harmonization. Generally, electricity markets operate on different geographical and time levels. Electricity can be traded from real-time (i.e., balancing markets) to long-term perspective (e.g., futures markets) and on several perimeters. Wholesale gas markets can also be divided according to their time perspective. The very long-term trade is organized through bilateral contracts, which are negotiated between suppliers and shippers. In addition to that, organized markets are in place. Trading times differ between years, quarters, months, weeks or on short-term in day-ahead and intra-day. The heat market is strongly seasondependent. Due to potential network losses, heat is characterized as a local commodity leading to a shorter and local value chain. Very often (district) heating systems are organized as monopolies, i.e. there is mostly one supplier of heat and several consumers. In the heat sector, there are generally no "organised" markets as such, even though, some sorts of heat market mechanisms can be found involving a day ahead planning and intra-day adjustments between the heat producers and the operator of the mechanism, like for the integrated heat market implemented in the Greater Copenhagen area in Denmark [2].

Several drivers point towards a need for more energy system integration, also at the market level. Firstly, increasing penetration of multi-carrier conversion technologies (e.g., combined heat and power (CHP), Natural gas fired plants (NGFPPs) and Power to gas (P2G) technologies) and storage technologies (e.g., thermal, mechanical, electro-chemical, chemical, electrical). Secondly, the increased ambition for local energy sufficiency and independency. Thirdly, recent changes specifically in gas markets and emerging trends to move from long term trading towards more short term trading. Finally, the increased uncertainty in electricity systems due to increased integration of variable RES (VRES) and the subsequent need for extra flexibility in the electricity system (and markets). A better and more explicit coupling of energy carrier markets can allow to leverage additional flexibility from market participants and to improve the global efficiency of the related energy systems.

\section{B. Literature Review}

The drivers mentioned in the previous section, strengthen the need for interdisciplinary research on coordinating the operation of integrated multi-energy systems and their markets. MES are energy systems with two or more energy carriers involved [3]. One can categorize the work in the literature on the subject in two groups: multi-energy system modeling, and (market based and non-market based) multi- 
energy scheduling. The former has received more attention than the latter in literature.

MES modeling includes studying the physical systems as well as the carrier flows over transmitting media [4]. The goal there is to investigate algorithms that enable greater coordination and co-optimization of systems of different carriers. Several articles have focused on the coordination between gas and electricity. For instance, reference [5] introduced an integrated gas and electricity model that considers the correlation between electricity supply from renewables (e.g., wind) and demand through conversion technologies (e.g., power-to-gas units) and their impact on co-ordinated operation of gas and power systems. Likewise, reference [6] investigates the effect of demand response on the operation of the two systems. A more detailed model is proposed in [7] to investigate the effects of variations in pipeline gas pressure on the ramp rates of natural gas fired power plants (NGFPPs).

Regarding studying the flows, authors in [8]-[10] propose a joint model to analyze and co-optimize gas and electricity flows in the respective networks. To this end, references [11], [12] proposed linearization techniques to study gas and flow in steady-state. Using the first principle of partial differential equations of a gas flow model, [13] studies the benefit of a more accurate gas model that considers the dynamics of gas flows on co-optimization of gas and power systems.

Other work studies the co-ordination in scheduling of MES considering their inter-dependencies. Neglecting the gas flow physics, reference [14] proposed a two-stage stochastic optimization framework to investigate the unit-commitment problem considering natural gas supply uncertainties. Following this work, Liu et al. proposed a bi-level optimization model that seeks to minimize the electricity production costs subject to natural gas feasibility constraints [15] and investigate coordinated scheduling of natural gas and power systems from the perspective of a joint operator [16].

Some recent work also investigates the operation of a joint multi-carrier energy market. Reference [17] investigates the participation of a NGFPP in a competitive electricity market with large share of renewables and volatile prices. Morais et al [18] look into the effect of natural gas and electricity pricing on investment planning regarding generation units. Yazdani-Damavandi et. al [4] investigate the impact of strategic behaviour of a strategic multi-energy participant (MEP) in the wholesale electricity market using an interactive aggregation model. Zhao et al. [19] propose a coordination mechanism to model day-ahead market clearing scheduling of natural gas and power that are operated by different entities. The proposed model considers physical properties of both systems and accurately reflects natural gas-flow transit. A key feature of the model is that it requires only limited information exchange between the gas and electricity markets (namely, fuel prices, supply and demand information) while keeping the network data and customer information for each system confidential.

Wang et al. in [20] study strategic behaviour of strategic participants in a synchronized integrated gas and electricity market that is operated by a single entity and allows for bidirectional gas-power tradings. The synchronized mechanism is shown to be more efficient compared with the sequential clearing mechanism that is in practice to date.

Along with larger integration of CHP units in the energy systems, the interaction between heat and electricity energy systems and markets have received increasing attention in the literature. Reference [21] assumes a local heat market and considers CHP units that participate in both power and heat markets. They model the economic dispatch problem of CHPs as a stochastic optimization framework. A CHP with varying power to heat profile is considered in [22] in a dynamic optimization model.

A number of works investigate the mutual interactions between the heat, electricity and gas systems. Reference [22], [23] introduce auction based mechanisms as a platform to solve an economic dispatch problem and trade heat, power and gas via a district energy operator.

\section{Contribution and organization}

The literature mentioned above which focuses on market based multi- energy scheduling, investigates the mutual influences of heat, natural gas and electricity (power) systems and/or markets, considering a simple integration level between the markets of different carriers. However, less attention has been paid to the different possibilities for integration and co-ordination between markets of different carriers and the plausible organizational and operational implications thereof on different stakeholders.

In this article we will introduce multi-carrier market schemes. A multi-carrier market scheme comprises a set of sub-markets to trade different energy carriers. A sub-market is assumed to be operated by one market operator who is responsible for the market clearing of this sub-market according to a specific objective. A sub-market is in effect a market on itself. In this analysis we will limit ourselves to day-ahead energy markets although the concept can easily be expanded to intra-day as well, and we will consider three different carriers, i.e. heat, electricity and gas.

The rest of this report is organized as follows. Section II introduces the five market schemes we study in the context of this work and reviews the main assumptions the market schemes proposed are based on. Section IV defines three indicators that we use to compare the performance of the proposed market schemes qualitatively. Finally, Section V concludes the paper and proposes some future research.

\section{ASSUMPTIONS AND MARKET DIMENSIONS}

Multi-carrier market schemes can be described by two market dimensions, i.e. the multi-carrier market integration and the locality of the sub-markets.

An important property of multi-carrier markets is that there are linkages among different carriers. This implies that the price of one carrier market should be calculated considering how it can affect and is affected by the prices of the other 
carrier markets [24]. Market linkages between carriers can be implicit or explicit [25].

An implicit linkage causes the market clearing price or a product price in one market to influence the price of a product in another market, without the existence of a formal constraint to enforce this link. This means that market participants have to predict or guess the effect of these linkages when they want to participate to the markets of different carriers.

When explicit links are introduced, different markets can be merged into a multi-product market, in our case a multi-carrier market. When considering multi-carrier market integration, we make a distinction between single carrier markets and multi-carrier markets. In a single carrier market, the interdependencies (i.e., linkages) between different carriers are not considered in the orders nor the clearing process, while in a multi-carrier market dependencies (i.e., linkages) between various carriers are explicitly considered in the market products and the clearing process. In effect, a multi-carrier market captures the dependencies between carriers through multicarrier order types (e.g., advanced orders which comprise a set of constraints that reflect how an order in one carrier (e.g., electricity) is dependent on the market clearing price and/or quantity of another carrier (e.g., heat and/or gas)).

Multi-carrier markets can cover several geographical territories that contain one or more energy systems from different carriers. The geographical scope of a market of gas and/or electricity can easily span over the geographical scope of one or several local market(s) of another carrier (e.g., heat). Electricity markets in Europe have been developing towards national and even international levels. Recently, there is also a trend in developing local electricity markets to enable competition at the distribution grid scale (regional or district level) due to the emergence of distributed energy resources (DERs) and energy storage systems. It is reasonable to assume that in the future, local electricity trades could also be settled through some type of local market arrangements. District heating and local multi-energy systems (MES) are typically localized. That is, they are not geographically extensive due to the economics (e.g. high investment) and high transport losses. As a result, they are typically developed around urban centres. Multi-carrier markets (associated to localized multienergy systems) can therefore be considered at this very local level, i.e. at the level of a local multi-energy system. On the other hand, multi-carrier systems and markets can also be considered at a higher (national or even supra-national) level. We therefore look into market schemes where one or more national or supra-national market (called global market in this report) overlaps (geographically and economically) with several local ones (called local markets). In what follows we make the following distinction: a global market is operated by a global market operator which manages energy trades at large regional-wide scale (e.g., national, supra-national) (mostly over high-voltage electricity network or high-pressure gas pipeline system); a local market is operated by a local market operator which manages energy trades at smaller local geographical scales comprising for example one or multiple medium or low voltage electricity network(s), low pressure gas network(s) and/or heat network(s).

Local electricity and gas markets are not considered in this article as they are not commonly deployed, although they could be an interesting track for future research. Single-carrier electricity and gas markets are thus only considered at the global level. On the other hand, local heat markets and local multi-carrier markets are considered as district heating systems and multi-energy systems are typically embedded locally. Global (single carrier) heat markets are not considered for the same reason. Multi-carrier systems and markets can also be considered at a higher (national or even supra-national) level. Two types of global multi-carrier markets are considered: an integrated market for electricity, heat and gas or one for electricity and gas only.

\section{MULTI-CARRIER MARKET SCHEMES}

In this section, we will introduce five multi-carrier market schemes under consideration which are visualized in Figure 1.

The first scheme, the Single carrier energy market scheme $(M S 1)$, resembles the existing energy market design in the EU the most. It anticipates the evolution of day-ahead gas markets to call markets with higher liquidity and the emergence of dayahead heat markets. This market scheme can be considered in some way as the current state with the addition of day ahead heat markets in the local context and the harmonization of the different day-ahead markets. In this scheme, the developments of the different markets are however projected to happen independently. As a result, separate (single) day-ahead energy markets are organized for the different energy carriers: there is no formal link between markets in the product definition and/or the market clearing process. Gas and electricity markets are considered as global markets and heat markets are local, as explained before. In the Single carrier market scheme, each market has its own gate closure time. By choosing adequate gate closure times, clearing times and publication times of the market results, these markets can in effect be sequential, thereby allowing market participants to readjust their positions for the next market(s), taking into account the clearing outcome of the previous market(s). In any case, market participants have to predict or guess certain interactions between carriers and market outcomes when submitting their orders in the single markets of the different carriers.

The second scheme, the Mixed single and multi-carrier energy market scheme (MS2), can be seen as an extension of the first market scheme where energy carrier market integration is considered at the local MES level. In this market scheme, there are multi-carrier markets for electricity, gas and heat at the local level. Interactions between carriers on the local multi-carrier markets are reflected via multi-carrier orders and therefore, are accounted for in the market clearing process. At the global level, there are only single carrier markets for electricity and gas. There is no integration between markets at local level and/or global level as they are pursuing their individual objectives (which may or may not be in conflict). Also here, a sequential set-up can be considered, e.g. where 


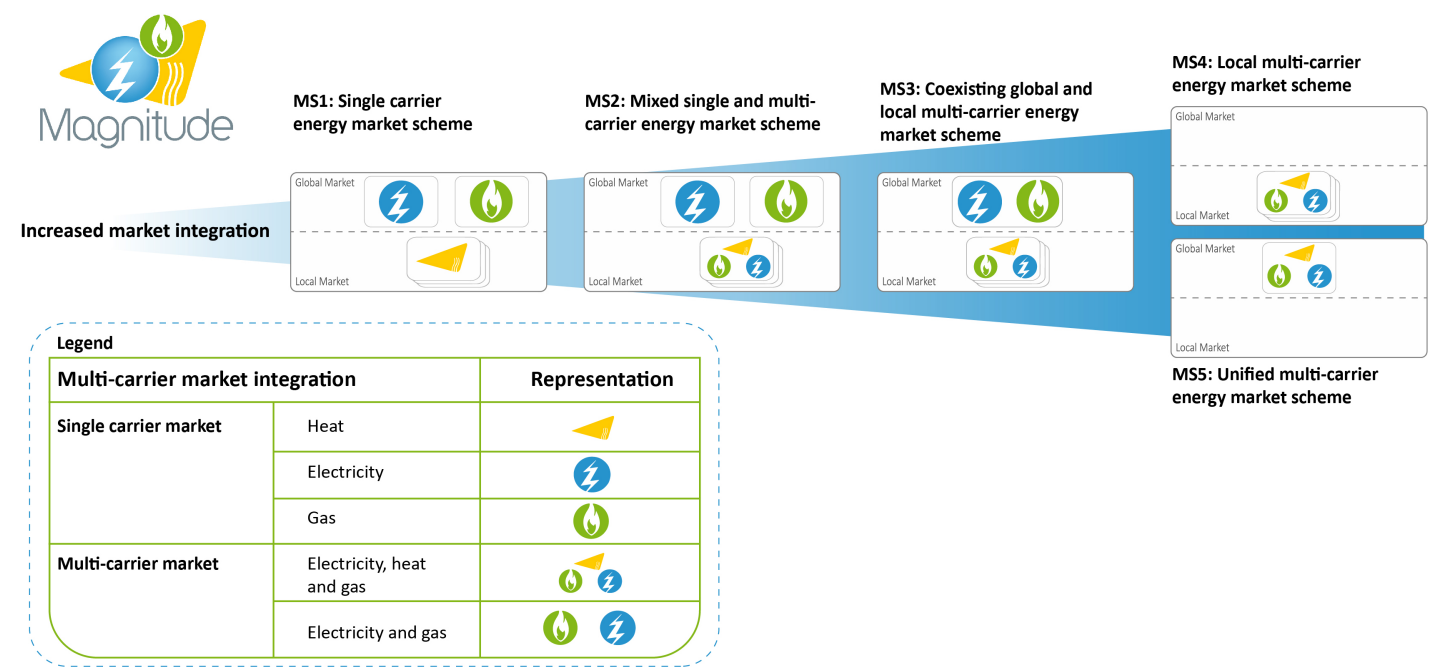

Figure 1. Visualization of the five market schemes. Moving from left to right, one can see that the level of integration increases and so the level by which inter-dependencies existing between different energy carrier systems are accounted for in a more pronounced manner in the market scheme and the clearing process.

first the local markets and afterwards the global markets are cleared (different sequences possible).

The third scheme Coexisting global and local multi-carrier energy market scheme (MS3), applies market integration of different energy carriers both at the local and global level. This market scheme could be seen as a logical next step after the second scheme: the main difference is that, here, gas and electricity markets at the global level are also integrated into a common multi-carrier market. Note that, markets at local and global levels are not integrated and therefore, each can pursue their own objective. As a result, in this market scheme there is a unique multi-carrier market for electricity and gas at the global level and there are multiple local multi-carrier markets for heat, gas and electricity at the local level. Interactions between carriers on multi-carrier markets are enabled via multi-carrier orders and constraints. Here too a sequential setup could be considered.

The fourth Market Scheme, the Local multi-carrier energy market scheme (MS4) can be considered as the most innovative scheme as there are only local multi-carrier markets (for heat, gas and electricity). Interactions between carriers on the local multi-carrier markets are considered via multi-carrier orders and interactions between the different local markets will also be considered. There are many possibilities for interactions between local markets. One set-up could be where a local multi-carrier market operator communicates with the other local multi-carrier market operators during the market clearing, so as to reach consensus on the physical trades between their corresponding market areas. However, such an approach would not allow considering strategic behaviors of the local market operators and might be limited by the problem size.

Finally, the fifth market scheme is the Unified multi-carrier energy market scheme (MS5). In this case there is one common market for the different energy carriers, operated by a unique multi-carrier Market Operator that processes orders at local and global levels. Interactions between carriers are considered via multi-carrier orders and constraints, and within the market clearing. In this specific market set-up, locality of the carriers needs to be explicitly accounted for in the market clearing.

\section{Qualitative EVAluation}

In this section Key Performance Indicators (KPIs) are defined, which can be used to compare the various market schemes. More specifically, we will compare the five market schemes based on the following three indicators: a) Market efficiency, measured through social welfare or surplus, the sum of economic surpluses across all market parties; b) Resemblance to the current EU energy market designs, defined as the extent to which the proposed multi-carrier market scheme resembles the current market across the EU regarding legal, economic, and administrative aspects; c) Confidentiality level, defined as the level of detail of information about technical and economic constraints of the underlying portfolio of the market participant shared with the market operator.

Figure 2 provides a qualitative evaluation of the five Multicarrier market schemes as discussed in section III based on the KPIs introduced above. It should be noted that these market schemes are very high-level and the scoring should therefore be interpreted as a relative and approximate comparison between different schemes.

Within the Unified multi-carrier energy market scheme (MS5) global maximisation of social welfare is possible. Therefore, market scheme 5 is considered as the benchmark to assess the performance of the other market schemes regarding market efficiency because it will give the highest social welfare. In the Single carrier energy market scheme (MS1), market participants have to predict or guess the market outcome when bidding with conversion technologies into the different markets, that is to internalize market risks via bidding strategies lowering both real and declared social welfare, and 


\begin{tabular}{|l|c|c|c|c|c|}
\hline \multirow{2}{*}{ Indicators } & \multicolumn{4}{|l|}{ Market Scheme } \\
\cline { 2 - 6 } & MS1 & MS2 & MS3 & MS4 & MS5 \\
\hline Social welfare & $\bullet$ & $\bullet$ & $\bullet$ & $\bullet$ & $\bullet$ \\
\hline Confidentiality level & $\bullet$ & $\bullet$ & $\bullet$ & $\bullet$ & $\bullet$ \\
\hline $\begin{array}{l}\text { Resemblance to the current EU } \\
\text { energy market designs }\end{array}$ & $\bullet$ & $\bullet$ & $\bullet$ & $\bullet$ & $\bullet$ \\
\hline$\bullet$ Highest $\bullet$ Higher $\bullet$ Moderate $\bullet$ Lower $\bullet$ Lowest \\
\hline
\end{tabular}

Figure 2. Qualitative KPI assessment for the five Market schemes

therefore, it will lead to social welfare values that are lower than the benchmark scheme. Also, in market schemes 2 to 4 , there are different markets, pursuing their own objective, but as one or more multi-carrier markets are present in each of these schemes, these schemes already allow to better capture linkages between different carriers. They are expected to have a higher market efficiency, compared to market scheme 1 and a lower market efficiency compared to market scheme 5. At this stage, we will not make a distinction between the three schemes (MS2, MS3, MS4) regarding market efficiency as specific design aspects, and possibly physical characteristics of the considered energy systems, would determine their respective ranking.

Regarding the second KPI, Resemblance to the current EU energy market designs, the Single carrier energy market scheme $(M S 1)$ is the closest to the current market structure that is implemented in Europe as markets for different carriers are dealt with separately and their linkages are not considered in the market clearing. Within the Mixed single and multicarrier energy market scheme (MS2), energy carrier market integration is considered only at the local level as this market scheme starts from the assumption that multi-carrier market integration will start at the local MES level. Therefore, it is assumed to be the second closest to the current energy market design. As multi-carrier market integration is present both at the local and global level in the Coexisting global and local multi-carrier energy market scheme (MS3), it is considered to be even further from the current design compared to the previous two schemes. In the Unified multi-carrier energy market scheme (MS5), there is only a global multi-carrier market which entails that all local heat trades need to be dealt with at the global level, so this entails a whole new approach so this diverges even more. Finally, the Local multi-carrier energy market scheme (MS4) is the furthest from the current design as only local markets are present, disregarding the existence of global markets as they exist today.

Finally, as multi-carrier markets are present in market schemes 2 to 5 and therefore at least some information on linkages between carriers will have to be shared with the multicarrier market operator, we assume that the confidentiality level of these schemes is lower than the one of market scheme 1 where there are only separate single-carrier markets. Again, we will not make a distinction between market schemes 2 to 5 regarding their difference in confidentiality level, as specific design aspects would determine their respective ranking.

\section{CONCLUSION}

In the current market setup, opportunities for price arbitrage among the energy carriers have already arisen. Even in the current circumstances, the market prices of the energy carriers influence each other, but multi-carrier energy systems cannot be fully integrated in the current energy system economics without proper multi-carrier energy market design.

Therefore, in this paper, we have proposed possible multicarrier market schemes, which allow to better reflect the interactions between the different energy carriers. The schemes range from a single carrier energy market scheme with separate, sequential day-ahead markets for different energy carriers to a unified multi-carrier energy market scheme with one unique multi-carrier market. Some advantages and disadvantages have been given for each of the schemes based on a qualitative KPI assessment.

In a work in preparation, we will investigate the development of a number of market designs that are plausible for the different multi-carrier schemes. This includes defining order types and clearing principles and studying organizational implications of implementing every design both analytically and numerically.

\section{ACKNOWLEDGMENT}

This work is part of the MAGNITUDE project which has received funding from the European Union's Horizon 2020 research and innovation programme under grant agreement No 774309 .

\section{REFERENCES}

[1] M. Geidl, "Integrated modeling and optimization of multi-carrier energy systems," Ph.D. dissertation, ETH Zurich, 2007.

[2] J. Boldt, "The district heating system in greater copenhagen area - in a free power market," 2018, greater Copenhagen Utility.

[3] T. Krause, G. Andersson, K. Frohlich, and A. Vaccaro, "Multipleenergy carriers: modeling of production, delivery, and consumption," Proceedings of the IEEE, vol. 99, no. 1, pp. 15-27, 2011.

[4] M. Yazdani-Damavandi, N. Neyestani, M. Shafie-khah, J. Contreras, and J. P. Catalao, "Strategic behavior of multi-energy players in electricity markets as aggregators of demand side resources using a bi-level approach," IEEE Transactions on Power Systems, vol. 33, no. 1, pp. 397-411, 2018.

[5] S. Chen, Z. Wei, G. Sun, K. W. Cheung, and Y. Sun, "Multi-linear probabilistic energy flow analysis of integrated electrical and naturalgas systems," IEEE Transactions on Power Systems, vol. 32, no. 3, pp. 1970-1979, May 2017.

[6] H. Cui, F. Li, Q. Hu, L. Bai, and X. Fang, "Day-ahead coordinated operation of utility-scale electricity and natural gas networks considering demand response based virtual power plants," Applied Energy, vol. 176, pp. 183 - 195, 2016. [Online]. Available: http://www.sciencedirect.com/science/article/pii/S030626191630589X

[7] Y. Zhou, C. Gu, H. Wu, and Y. Song, "An equivalent model of gas networks for dynamic analysis of gas-electricity systems," IEEE Transactions on Power Systems, vol. 32, no. 6, pp. 4255-4264, Nov 2017.

[8] B. Zhao, A. J. Conejo, and R. Sioshansi, "Unit commitment under gas-supply uncertainty and gas-price variability," IEEE Transactions on Power Systems, vol. 32, no. 3, pp. 2394-2405, 2017.

[9] A. Martinez-Mares and C. R. Fuerte-Esquivel, "A unified gas and power flow analysis in natural gas and electricity coupled networks," IEEE Transactions on Power Systems, vol. 27, no. 4, pp. 2156-2166, 2012.

[10] Z. Qiao, S. Huang, R. Li, Q. Guo, H. Sun, and Z. Pan, "Unified power flow analysis in natural gas and electricity coupled networks considering the uncertainty of wind power," Energy Procedia, vol. 103, pp. 322-327, 2016. 
[11] C. M. Correa-Posada and P. Sánchez-Martın, "Security-constrained optimal power and natural-gas flow," IEEE Transactions on Power Systems, vol. 29 , no. 4 , pp. 1780-1787, 2014.

[12] C. Shao, X. Wang, M. Shahidehpour, X. Wang, and B. Wang, "An milp-based optimal power flow in multicarrier energy systems," IEEE Transactions on Sustainable Energy, vol. 8, no. 1, pp. 239-248, 2017.

[13] A. Zlotnik, L. Roald, S. Backhaus, M. Chertkov, and G. Andersson, "Coordinated scheduling for interdependent electric power and natural gas infrastructures," IEEE Transactions on Power Systems, vol. 32, no. 1, pp. 600-610, 2017.

[14] C. Liu, M. Shahidehpour, Y. Fu, and Z. Li, "Security-constrained unit commitment with natural gas transmission constraints," IEEE Transactions on Power Systems, pp. 1523-1536, 2009.

[15] C. Liu, M. Shahidehpour, and J. Wang, "Coordinated scheduling of electricity and natural gas infrastructures with a transient model for natural gas flow," Chaos: An Interdisciplinary Journal of Nonlinear Science, vol. 21, no. 2, p. 025102, 2011. [Online]. Available: https://doi.org/10.1063/1.3600761

[16] C. Liu, "Application of augmented lagrangian relaxation to coordinated scheduling of interdependent hydrothermal power and natural gas systems," IET Generation, Transmission \& Distribution, vol. 4, pp. 13141325(11), December 2010.

[17] P. Dueñas, T. Leung, M. Gil, and J. Reneses, "Gas-electricity coordination in competitive markets under renewable energy uncertainty," IEEE Transactions on Power Systems, vol. 30, no. 1, pp. 123-131, Jan 2015.

[18] M. S. Morals and J. W. M. Lima, "Natural gas network pricing and its influence on electricity and gas markets," in 2003 IEEE Bologna Power Tech Conference Proceedings, vol. 3, June 2003, pp. 6 pp. Vol.3-.

[19] B. Zhao, A. Zlotnik, A. J. Conejo, R. Sioshansi, and A. M. Rudkevich, "Shadow price-based co-ordination of natural gas and electric power systems," IEEE Transactions on Power Systems, 2018.

[20] C. Wang, W. Wei, J. Wang, F. Liu, and S. Mei, "Strategic offering and equilibrium in coupled gas and electricity markets," IEEE Transactions on Power Systems, vol. 33, no. 1, pp. 290-306, Jan 2018.

[21] L. Mitridati and P. Pinson, "Optimal coupling of heat and electricity systems: A stochastic hierarchical approach," in 2016 International Conference on Probabilistic Methods Applied to Power Systems (PMAPS). IEEE, 2016, pp. 1-6.

[22] D. Xie, Y. Lu, J. Sun, C. Gu, and G. Li, "Optimal operation of a combined heat and power system considering real-time energy prices," IEEE Access, vol. 4, pp. 3005-3015, 2016.

[23] W. Zhong, K. Xie, Y. Liu, C. Yang, and S. Xie, "Auction mechanisms for energy trading in multi-energy systems," IEEE Transactions on industrial informatics, vol. 14, no. 4, pp. 1511-1521, 2018.

[24] S. A. Gabriel, A. J. Conejo, J. D. Fuller, B. F. Hobbs, and C. Ruiz, Complementarity modeling in energy markets. Springer Science \& Business Media, 2012, vol. 180.

[25] S. Stoft, "Power system economics," Journal of Energy Literature, vol. 8, pp. 94-99, 2002. 